\title{
Anti-metabolic syndrome and immunostimulant activities of Egyptian fenugreek seeds in diabetic/obese and immunosuppressive rat models
}

\author{
Gamal Ramadan*, Nadia M. El-Beih and Hanaa F. Abd El-Kareem \\ Zoology Department, Faculty of Science, Ain Shams University, Abbasseya 11566, Cairo, Egypt \\ (Received 9 August 2010 - Revised 12 October 2010-Accepted 14 October 2010 - First published online 23 December 2010)
}

\section{Abstract}

Preliminary trials have suggested possible hypoglycaemic, hypolipidaemic and immunomodulatory properties of the fenugreek plant Here, we evaluated and compared the efficacy of Egyptian fenugreek seed powder (FSP, 0.5 and $1.0 \mathrm{~g} / \mathrm{kg}$ body weight) in alleviating the experimentally induced metabolic syndrome (in type 1 diabetic and obese rat models) and experimentally induced immunosuppression and delay in burn-healing (in cyclophosphamide (CP)-treated rats). FSP significantly alleviated $(P<0 \cdot 05-0 \cdot 001)$ most signs of the metabolic syndrome resulting from experimentally induced type 1 diabetes and obesity by $40-76$ and $56-78 \%$, respectively, including hyperglycaemia, hyperlipidaemia, elevation in atherogenic indices, impairment of liver functions, severe changes in body weight and oxidative stress. Besides, FSP (especially the high dose) completely modulated the immunosuppressive activity of CP including leucopenia (resulting from neutropenia and lymphopenia), decrease in weights and cellularity of lymphoid organs, serum $\gamma$-globulin level, delayed type of hypersensitivity response and delay in the skin-burning healing process. FSP decreased the immunosuppressive activity of CP by $57-108 \%$. These beneficial effects of FSP were dose dependent in most cases, and FSP doses used here were considered safe in general. FSP was more efficient in alleviating the signs of the metabolic syndrome in the obese animals (over 9\%) than in the type 1 diabetic animals. Moreover, the immunostimulant activity of fenugreek seeds exceeded their anti-metabolic syndrome activity by $15-24 \%$. In conclusion, fenugreek seeds may be useful not only as a dietary adjunct for the control of the metabolic syndrome in diabetic/obese patients, but also as an immunostimulant in immunocompromised patients such as those under chemotherapeutic interventions.

Key words: Albino rats: Diabetes and obesity: Fenugreek seeds: Immunosuppression: Metabolic syndrome

The present number of diabetics worldwide exceeds 150 million and is predicted to be $>300$ million by the year 2025 due to the increase in sedentary lifestyles, consumption of an energy-rich diet, obesity and higher life $\operatorname{span}^{(1)}$. Obesity has increased at an alarming rate in recent years and is now a worldwide health problem, since it is a major risk factor for insulin resistance or type 2 diabetes, impairment of liver functions and even cancer, for which the social costs are incalculable ${ }^{(2)}$. Cardiovascular complications (e.g. hypertension and coronary artery and peripheral vascular diseases) due to the metabolic syndrome, a clustering of pathological conditions including hyperglycaemia/dyslipidaemia (resulting from type 1 and 2 diabetes) and liver steatosis/dysfunctions, are a major cause of morbidity and mortality in diabetic and obese patients $^{(3)}$. Cyclophosphamide (CP) is an alkylating agent widely used to treat a variety of malignant diseases such as lymphoma, myeloma and chronic lymphocytic leukaemia $^{(4)}$. However, CP intake is associated with many serious side effects including immunosuppression and dyslipidaemia, which explains why an impressive part of the morbidity and mortality in cancer patients treated with such chemotherapeutic agents is caused by infections and hyperlipidaemic cardiomyopathy ${ }^{(5-7)}$. Free radicals, especially reactive oxygen species, are involved in the pathogenesis of the metabolic syndrome and $\mathrm{CP}$-induced immunosuppression $^{(6-8)}$.

Due to the harmful side effects of synthetic drugs, the enormous cost and the inability of existing modern therapies to control all pathological aspects of the diabetic disorder and obesity, and the poor availability of advanced therapies for many rural populations in developing countries, many dietary supplements that have antioxidant activity and can modulate glucose homeostasis, potentially improve lipid parameters, and are less toxic than Western medicine have been recommended for the treatment of diabetes mellitus and obesity as well as their complications nowadays ${ }^{(8-10)}$. In addition, a natural modulator that shows immunostimulant activity and can alleviate immunosuppressive side effects in CP-treated cancer patients is

Abbreviations: ALP, alkaline phosphatase; ASAT, aspartate aminotransferase; CP, cyclophosphamide; FSP, fenugreek seed powder.

*Corresponding author: Assistant Professor G. Ramadan, fax + 202 26842123, email gamal_ramadan@hotmail.com 
urgently needed ${ }^{(5,7)}$. Preliminary trials have suggested possible hypoglycaemic, hypolipidaemic and immunomodulatory properties of the fenugreek plant ${ }^{(9,11-14)}$. The present study aimed to evaluate and compare the efficacy of Egyptian fenugreek seed powder (FSP) in alleviating the experimentally induced metabolic syndrome (in two different animal models: type 1 diabetic and obese rat models) and immunosuppression as well as delay in burn-healing (for the first time, to our knowledge, in $\mathrm{CP}$ treated rats). Two different doses of FSP $(0.5$ and $1.0 \mathrm{~g} / \mathrm{kg}$ body weight) were used in the present study to test whether the obtained modulatory effects were dose dependent. Furthermore, the present study investigated any deleterious effects caused by consuming the Egyptian FSP.

\section{Materials and methods}

\section{Materials}

Alloxan monohydrate, cholesterol and CP (200 $\mathrm{mg}$ /ampoule) were purchased from Sigma-Aldrich (St Louis, MO, USA), WinLab (Market Harborough, Leicestershire, UK) and Baxter Oncology GmbH (Frankfurt, Germany), respectively. Egyptian fenugreek (Trigonella foenum-graecum L. cultivar Baladi, family: Leguminosae, known locally as helba) seeds were purchased from a local herbal store (SMA Trading Company, Giza, Egypt), kindly authenticated by Professor Dr Raifa A. Hassanein (Botany Department, Faculty of Science, Ain Shams University, Cairo, Egypt), and a voucher specimen was deposited in our department. Adult male Wistar albino rats (Rattus norvegicus), weighing 125-135g, were obtained from the National Research Centre in Giza, Egypt. Animals were housed in suitable cages and acclimatised to laboratory conditions for a period of 1 week before the commencement of the experiments. Rats were fed standard rodent food pellets (Agricultural-Industrial Integration Company, Giza, Egypt) and double-distilled water. The standard rodent food pellets contain wheat bran, dried clover, maize, bean hay, methionine, molasses, salt, in addition to mineral and vitamin mix. The amount of crude proteins, fats and fibres in the food pellets are 12, 2.4 and $14 \%$, respectively. The energy content of the standard diet is $920.48 \mathrm{~kJ} / 100 \mathrm{~g}$. All animals were humanely treated in accordance with the WHO guidelines for animal care, and the study design was approved by the Ain Shams University Research Ethics Committee.

\section{Experimental design and treatment schedule}

Experimental animals were randomly divided into twelve groups of five rats each: three healthy groups; three diabetic groups; three obese groups; three immunosuppressive groups. In the healthy groups, animals were treated by oral administration and daily with 0.5 or $1.0 \mathrm{~g} / \mathrm{kg}$ body weight (low or high dose, respectively) of FSP suspended in $1.0 \mathrm{ml}$ distilled water for 4 weeks. In the diabetic groups, animals were given the low or high dose of FSP orally and daily for 4 weeks and subcutaneously received an injection of alloxan ( $120 \mathrm{mg} / \mathrm{kg}$ body weight) for the first $3 \mathrm{~d}$ only to induce hyperglycaemia ${ }^{(8)}$. In the obese groups, animals were fed a hypercholesterolaemic diet (standard rodent food pellets plus $2 \%$ cholesterol, $\mathrm{w} / \mathrm{w})$ to induce hyperlipidaemia ${ }^{(8)}$ and given the low or high dose of FSP orally and daily for 4 weeks. In the immunosuppressive groups, animals were given the low or high dose of FSP orally and daily for 4 weeks and received a single intraperitoneal injection $(25 \mathrm{mg} / \mathrm{kg}$ body weight) of $\mathrm{CP}^{(7)}$ on day 24 (i.e. $6 \mathrm{~d}$ before killing the animals) to induce immunosuppression. The healthy control, alloxanonly-treated, cholesterol-only-treated and CP-only-treated groups received $1.0 \mathrm{ml}$ distilled water orally and daily, as a vehicle, instead of FSP suspension for 4 weeks.

\section{Blood and tissue sampling}

Animals were fasted overnight and subjected to light diethyl ether anaesthesia before killing on day 29. Blood was collected into clean test-tubes with or without EDTA. A portion of blood with EDTA $(0.5 \mathrm{ml})$ was used to measure the total and differential leucocyte counts by Coulter (Hemat 8 analyser; SEAC, Freiburg, Germany). Another portion of blood without EDTA was used to separate serum, which was divided into samples and preserved at $-70^{\circ} \mathrm{C}$ for further analysis. Immediately after killing the animals, the thymus and spleen were separated out of the body, cleaned and weighed. Bone marrow was also collected from the left femur bone. The cellularity of lymphoid organs was measured by a Neubauer counting chamber (Paul Marienfeld GmbH, Bad Mergentheim, Germany) after lysing the erythrocytes by red blood erythrocyte lysis solution (Applichem GmbH, Darmstadt, Germany). The liver was quickly perfused in situ (via the hepatic portal vein) with ice-cold saline (to remove erythrocytes and clots) and homogenised in cold buffer (0.1 M-phosphate buffer, $\mathrm{pH} 7 \cdot 4)$. Then, the homogenate was stored at $-70^{\circ} \mathrm{C}$ until used for the determination of GSH through the reaction of its sulfhydryl group with Ellman's reagent to give 5-thio-2-nitrobenzoic acid ${ }^{(15)}$.

\section{Measurements}

Food intake (on a per-group basis) was measured weekly. Body-weight gain or loss was calculated by the following equation: body-weight gain or loss = body weight at the end of the experiment - body weight at the beginning of the experiment. Serum glucose concentration was colorimetrically estimated using glucose oxidase and peroxidase $^{(16)}$. Serum total lipid concentration was chemically determined by the phosphovanillin method ${ }^{(17)}$. Serum total cholesterol ${ }^{(18)}$, TAG $^{(19)}$ and HDL-cholesterol ${ }^{(20)}$ concentrations were colorimetrically determined using 
peroxidase-coupled methods. Serum LDL-cholesterol concentration was calculated according to the equation of Friedewald et $a l^{(21)}$ : LDL-cholesterol $=$ total cholesterol - (TAG/5) - HDL-cholesterol. Atherogenic indices were calculated as follows: atherogenic index $(1)=$ total cholesterol:HDL-cholesterol ratio, atherogenic index (2) = LDL-cholesterol:HDL-cholesterol ratio. Serum alanine aminotransferase and aspartate aminotransferase (ASAT) activities were colorimetrically measured ${ }^{(22)}$. Serum alkaline phosphatase (ALP) activity was estimated from the rate of conversion of $p$-nitrophenylphosphate to $p$-nitrophenol ${ }^{(23)}$. Serum $\gamma$-globulin concentration was estimated by the Helena cellulose acetate protein electrophoresis as described previously ${ }^{(7)}$. The percentage of change of any parameter $=((T-C) / C) \times 100$, where $T$ is the mean value of the parameter in the treated group and $C$ is the mean value of the parameter in the control group.

\section{Delayed type of hypersensitivity response}

The delayed type of hypersensitivity response was determined as described previously ${ }^{(11)}$ with some modifications. Briefly, on day 29, animals were subcutaneously immunised with $2.25 \times 10^{10}$ sheep red blood corpuscles (Institute of the Agricultural Researches, Giza, Egypt). On the fifth day of immunisation, the animals were again challenged with $1 \times 10^{10}$ cells in the left hind footpad. The increase in footpad thickness was measured $24 \mathrm{~h}$ after the second challenge by vernier calliper (Samir and Ali Bookshop, Cairo, Egypt). The right footpad was injected with saline solution and served as the trauma control for non-specific swelling.

\section{Skin-burning healing process}

On day 29, hairs in the back of animals were shaved, and a skin burn was performed by a hot stamp under local dermatological anaesthetic spray (Lidocaine, The Arab Drug Company, Cairo, Egypt). Then, the healing process was followed as described previously ${ }^{(24)}$, and photos were taken periodically.

\section{Statistical analysis}

Data are presented as means with their standard errors. Statistical analysis was performed with one-way ANOVA or the Friedman test (only for comparing the percentages of changes of all parameters), and the differences among groups were determined by Bonferroni's or Dunn's multiple comparison test, respectively ${ }^{(25)}$, using GraphPad Prism version 4.03 for Windows (GraphPad Software, Inc., San Diego, CA, USA). $P$ values of $<0.05,<0.01$ and $<0.001$ were considered statistically significant, highly significant and very highly significant, respectively.

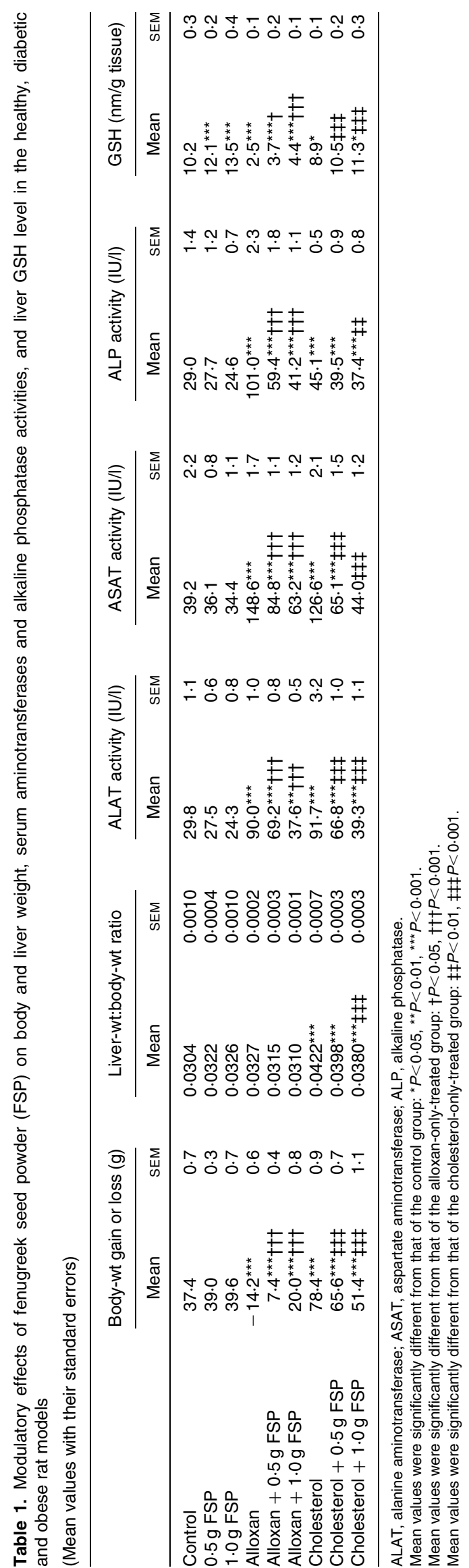




\section{Results}

Anti-metabolic syndrome efficacy of fenugreek seeds in the type 1 diabetic rat model

The present study showed that treatment of rats with alloxan alone did not significantly alter $(P>0.05$, $t=2 \cdot 761$, difference between means -0.0023 , 95\% CI $-0.0049,0.0003)$ the liver-weight:body-weight ratio, but induced a very highly significant increase $(P<0.001)$ in serum alanine aminotransferase (202\%), ASAT (279\%) and ALP (248\%) activities, glucose (226\%), total lipid (53\%), total cholesterol (46\%) and TAG (117\%) levels, and atherogenic indices (147-892\%) compared with the healthy control animals (Tables 1 and 2). On the other hand, it induced a very highly significant decrease $(P<0.001)$ in body weight $(31 \%)$ and liver GSH level (76\%) compared with the healthy control animals (Table 1). As shown in Tables 1 and 2, FSP significantly modulated all these harmful changes induced by alloxan (40-76\%, P<0.05-0.001 compared with the alloxanonly-treated group). These modulatory effects significantly increased by increasing the dose of FSP in all cases $(P<0 \cdot 001)$, except in the liver GSH and serum total cholesterol levels where no statistically significant difference in modulation $(P>0.05)$ was found between both doses of FSP (Tables 1 and 2). Food intake was not significantly changed $(P>0.05)$ in alloxan with/without FSP-treated groups compared with the healthy control group (data not shown).

The percentages of changes of all parameters measured in the alloxan-only-treated group and the groups treated with alloxan plus either the low or high dose of FSP were 154.9 (SEm 70.7, $P<0.001$ ), 93.1 (SEm 49.3, $P<0.05$ ) and 37.7 (SEM $24 \cdot 4, P>0.05$ ), respectively, compared with the healthy control group. All of these results revealed that the most modulatory effects on diabetic rats were induced by the high dose of FSP.

\section{Anti-metabolic syndrome efficacy of fenugreek seeds in the obese rat model}

The present study showed that feeding rats a hypercholesterolaemic diet induced a very highly significant increase $(P<0.001)$ in the body weight $(25 \%)$, liverweight:body-weight ratio (39\%), serum alanine aminotransferase (208\%), ASAT (223\%) and ALP (56\%) activities, glucose (63\%), total lipid (75\%), total cholesterol (139\%) and TAG (113\%) levels, and atherogenic indices (203-1554\%) compared with the healthy control animals (Tables 1 and 2). On the other hand, it induced a significant decrease $(13 \%, P<0 \cdot 05, t=3 \cdot 712$, difference between means $1.234,95 \%$ CI $0.189,2 \cdot 279)$ in the liver GSH level compared with the healthy control animals (Table 1). As shown in Tables 1 and 2, FSP significantly modulated all these harmful changes induced by the hypercholesterolaemic diet $(56-78 \%, P<0 \cdot 01-0 \cdot 001$

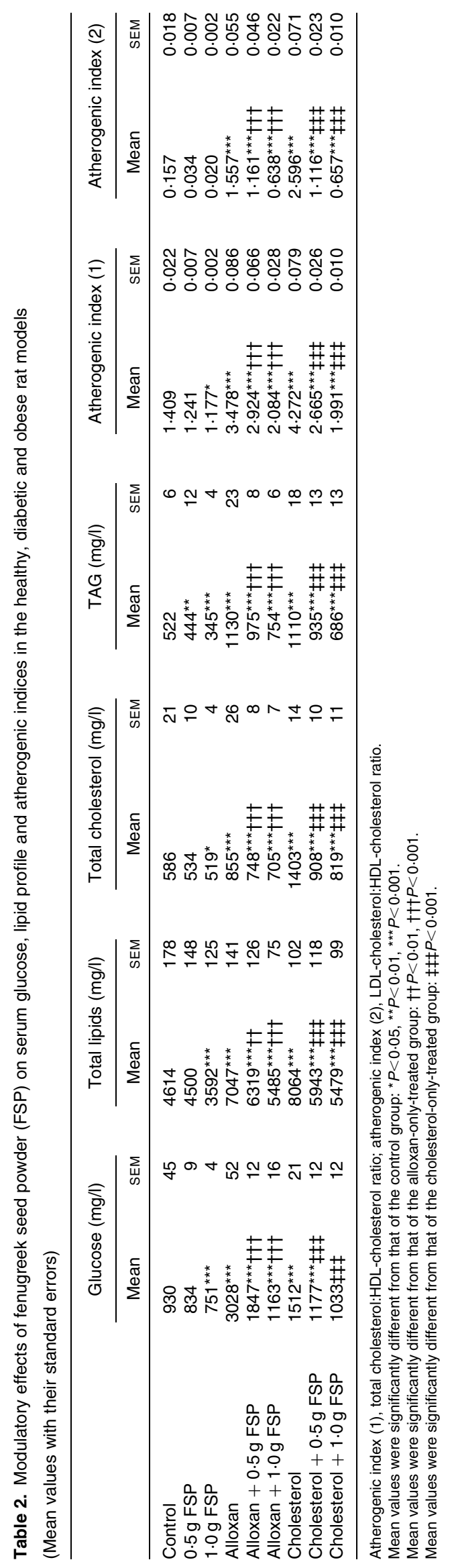


compared with the cholesterol-only-treated animals), except that the low dose of FSP did not significantly change $(P>0.05)$ the increase in the liver-weight:bodyweight ratio and serum ALP activity shown in the cholesterol-treated animals. The modulatory effects significantly increased by increasing the dose of FSP in all cases ( $P<0 \cdot 01-0 \cdot 001)$, except in the liver-weight:body-weight ratio, serum ALP activity, and liver GSH and serum total lipid levels where no statistically significant difference in modulation $(P>0.05)$ was found between both doses of FSP (Tables 1 and 2). The liver GSH level was completely modulated in the group that received the hypercholesterolaemic diet plus the low dose of FSP $(P>0.05, t=1.041$, difference between means - 0.346, 95\% CI -1.391 , 0.699); moreover, it significantly increased by $11 \%$ above the normal level $(P<0.05, t=3.531$, difference between means $-1 \cdot 174,95 \%$ CI $-2 \cdot 219$, -0.129$)$ in the group that received the hypercholesterolaemic diet plus the high dose of FSP, compared with the healthy control group (Table 1). Serum ASAT activity and glucose level became comparable with those of the healthy control group $(P>0.05)$ in the group that received the hypercholesterolaemic diet plus the high dose of FSP only (Tables 1 and 2). Food intake was not significantly changed $(P>0.05)$ in the cholesterol with/without FSP-treated groups compared with the healthy control group (data not shown).

The percentages of changes of all parameters measured in the cholesterol-only-treated group and the groups treated with cholesterol plus either the low or high dose of FSP were 213.6 (SEM 113.6, $P<0.001$ ), 93.9 (SEM 44.2, $P<0.05$ ) and 46.5 (sem $23.0, P>0.05$ ), respectively, compared with the healthy control group. All of these results revealed that the most modulatory effects on obese rats were induced by the high dose of FSP. In addition, FSP was more efficient in alleviating the signs of the metabolic syndrome in the obese animals (over 9\%) than in the type 1 diabetic animals.

\section{Immunostimulant efficacy of fenugreek seeds in the immunosuppressive rat model}

The present study showed that treatment of rats with $\mathrm{CP}$ alone induced severe blood leucopenia $(P<0 \cdot 001$, $t=5 \cdot 2$, difference between means $2 \cdot 4$, $95 \%$ CI $1 \cdot 0,3 \cdot 8)$, which was mainly due to neutropenia $(P<0.001$, $t=4.923$, difference between means 1.483 , 95\% CI $0.565,2 \cdot 401)$ and lymphopenia $(P<0.05, t=3 \cdot 422$, difference between means 0.789, 95\% CI 0.087, 1.492), compared with the healthy control animals (Fig. 1). It also caused a significant decrease $(P<0.05-0.001)$ in weights $(25-35 \%)$ and cellularity (59-65\%) of lymphoid organs as well as serum $\gamma$-globulin level (51\%) and delayed type of hypersensitivity response (48\%) compared with the healthy control animals (Figs. 2 and 3). Moreover, it caused a delay in the skin-burning healing process by prolonging the inflammatory phase (swelling, redness and warmth) and the proliferative phase (mucus secretion and pellicle detachment), as shown on days 18 and 26 in the CP-only-treated animals $v$. days 8 and 18 in the healthy control animals, respectively (Fig. 4). The remodelling phase, the final phase where the intact skin replaced the burning one, the diameter of the burn decreased until complete recovery and the hairs grew again, started/ended on day 33/41 in the CP-only-treated animals $v$. day 26/33 in the healthy control animals, respectively. As shown in Figs. 1-4, both FSP doses completely modulated all immunosuppressive activities of CP $(P>0.05$ and $P<0.05-0.001$ compared with the healthy control animals and CP-onlytreated animals, respectively), except that the low dose of FSP partially modulated the decrease in the cellularity of lymphoid organs and serum $\gamma$-globulin level as well as the delay in the skin-burning healing process shown in the CP-treated animals $(P<0 \cdot 05-0.001$ compared with the healthy control animals). The immunosuppressive activity of CP decreased by $57-108 \%$ in the FSP-treated animals. The modulatory effects on the spleen-weight: body-weight ratio and the cellularity of lymphoid organs (a)

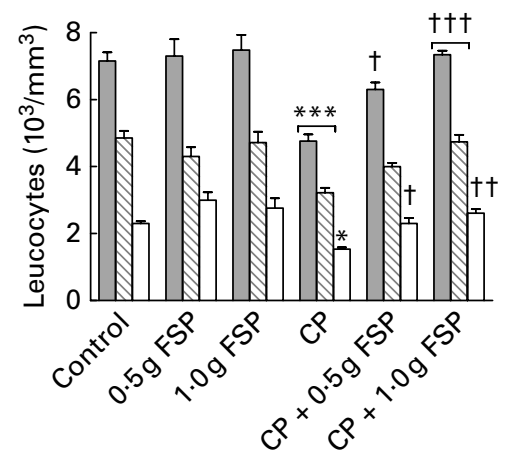

(b)

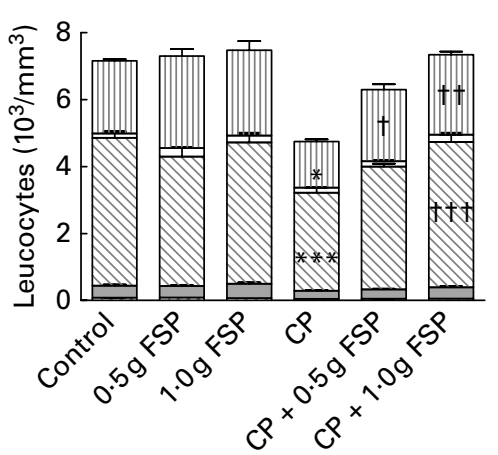

Fig. 1. Modulatory effects of fenugreek seed powder (FSP) on the changes in blood (a) total and (b) differential leucocyte counts in the healthy and immunosuppressed rat models. Values are means, with standard errors represented by vertical bars. Mean values were significantly different from that of the control group: ${ }^{\star} P<0.05$, ${ }^{\star \star \star} P<0.001$. Mean values were significantly different from that of the cyclophosphamide (CP)-only-treated group: $† P<0.05$, $\dagger \dagger P<0.01$,

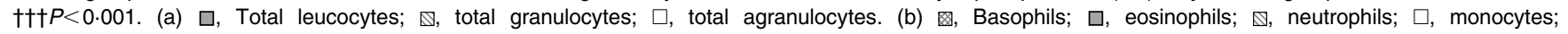
प, lymphocytes. 
(a)

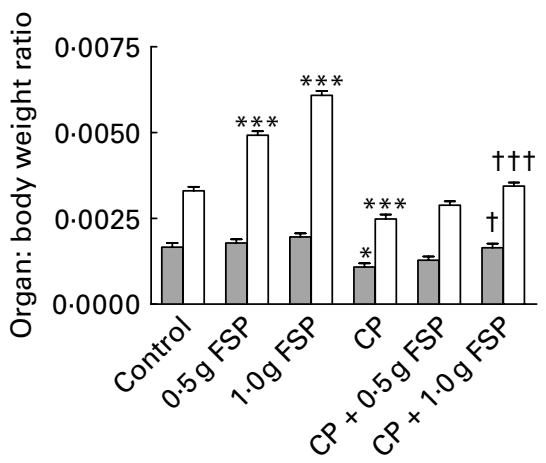

(b)

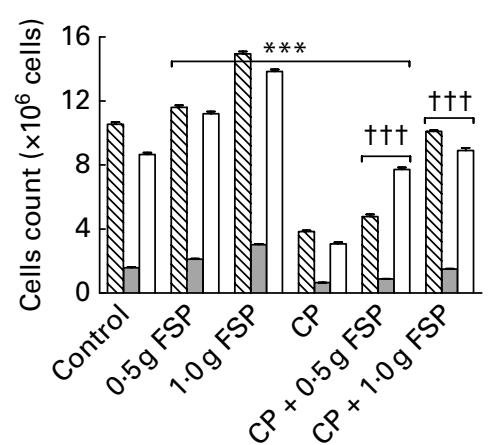

Fig. 2. Modulatory effects of fenugreek seed powder (FSP) on the changes in weights (a) and cellularity (b) of lymphoid organs in the healthy and immunosuppressed rat models. Values are means, with standard errors represented by vertical bars. Mean values were significantly different from that of the control group: ${ }^{\star} P<0.05$, ${ }^{\star \star \star} P<0.001$. Mean values were significantly different from that of the cyclophosphamide $(\mathrm{CP})$-only-treated group: $+P<0.05$,

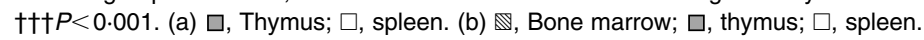

significantly increased ( $P<0.05$ and $<0.001$, respectively) by increasing the dose of FSP (Fig. 2).

The percentages of changes of all parameters measured in the CP-only-treated group and the groups treated with CP plus either the low or high dose of FSP were -38.6 (sem 5.0, $P<0.001$ ), -16.7 (SEM 5.5, $P>0.05$ ) and 2.9 (sEm $6 \cdot 0, P>0.05$ ), respectively, compared with the healthy control group. All of these results revealed that the most modulatory effects on immunosuppressive rats were induced by the high dose of FSP. In addition, the modulatory effects of FSP on CP-induced immunosuppression exceeded that on alloxan-induced type 1 diabetes and cholesterol-induced obesity (24 and 15\%, respectively).

\section{Beneficial and deleterious effects caused by fenugreek seed consumption in healthy rats}

The present study showed that treatment of healthy rats with either the low or high dose of FSP alone significantly increased $(P<0 \cdot 001)$ the liver GSH level $(19-32 \%$; Table 1), the spleen-weight:body-weight ratio (49-84\%;
Fig. 2(a)) and the cellularity of lymphoid organs (10-91\%; Fig. 2(b)), accelerated the skin-burning healing process (Fig. 4) and significantly decreased $(P<0.01-$ 0.001) the serum TAG level (15-34\%; Table 2) compared with the healthy control animals. These beneficial effects significantly increased by increasing the dose of FSP in all cases $(P<0 \cdot 01-0 \cdot 001)$. On the other hand, only the high dose of FSP significantly decreased $(P<0.05-0.001)$ the serum glucose (19\%), total lipid (22\%) and total cholesterol (11\%) levels as well as the first atherogenic index (17\%), and significantly increased ( $47 \%, P<0 \cdot 01$, $t=4.492$, difference between means -0.061 , 95\% CI $-0 \cdot 102,-0 \cdot 020)$ the delayed type of hypersensitivity response in the healthy rats compared with the healthy control animals (Table 2 and Fig. 3). All other parameters measured in the present study did not significantly alter in the healthy rats by both FSP doses $(P>0.05$ compared with the healthy control animals). Moreover, food intake, serum total protein, albumin, globulin fractions $\left(\alpha_{1^{-}}, \alpha_{2^{-}}\right.$, $\beta$ - and $\gamma$-globulins) and bilirubin (total, direct and indirect) levels, erythrocyte and platelet counts, $\mathrm{Hb}$ content, packed

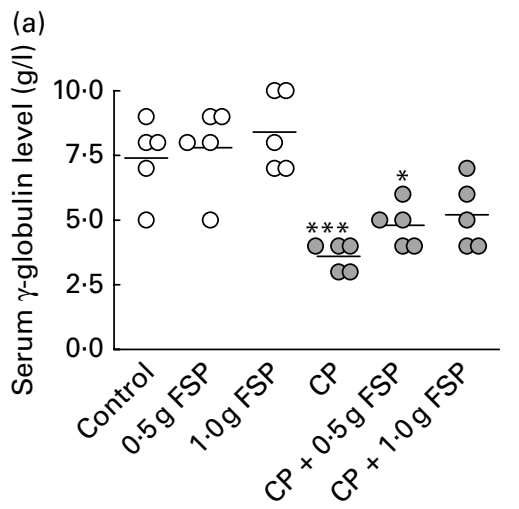

(b)

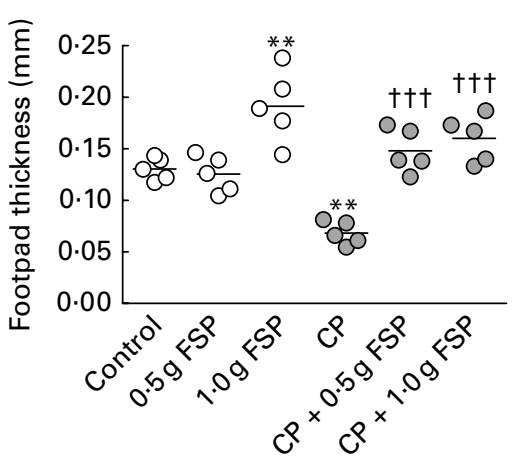

Fig. 3. Modulatory effects of fenugreek seed powder (FSP) on the serum $\gamma$-globulin level (a) and delayed type of hypersensitivity response (b) in the healthy and immunosuppressed rat models. Individual values are shown, with means represented by horizontal bars. Mean values were significantly different from that of the control group: ${ }^{\star} P<0.05$, ${ }^{\star \star} P<0.01,{ }^{* \star *} P<0.001$. Mean values were significantly different from that of the cyclophosphamide (CP)-only-treated group: $+\dagger+P<0.001$. 
(a)

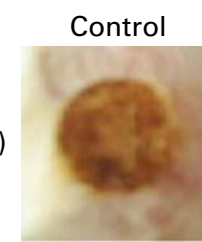

(b)

(c)
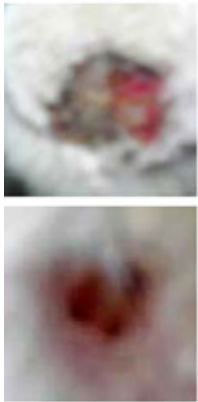

(d)

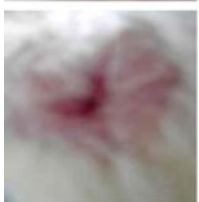

(e)

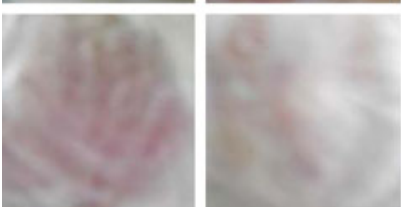

$0.5 \mathrm{~g}$ FSP
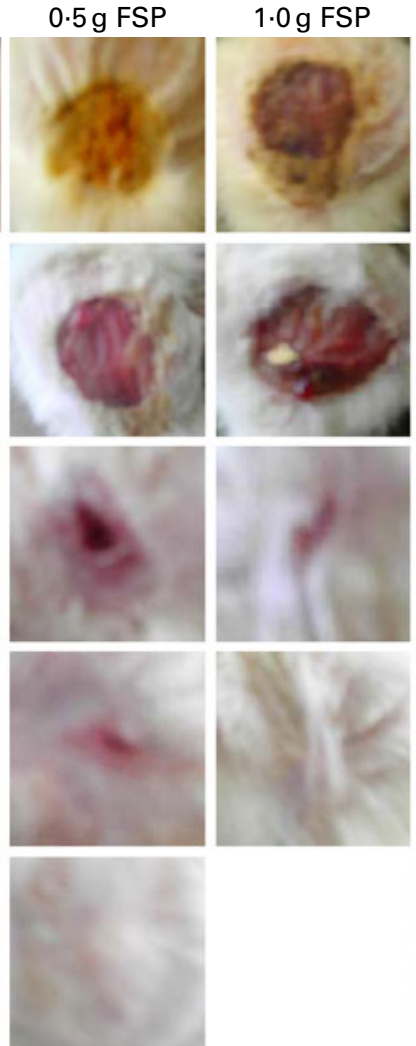
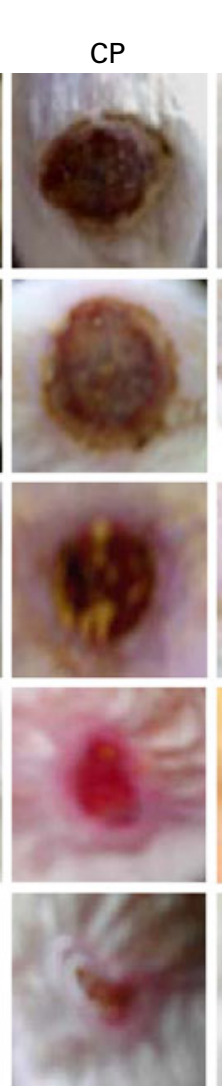
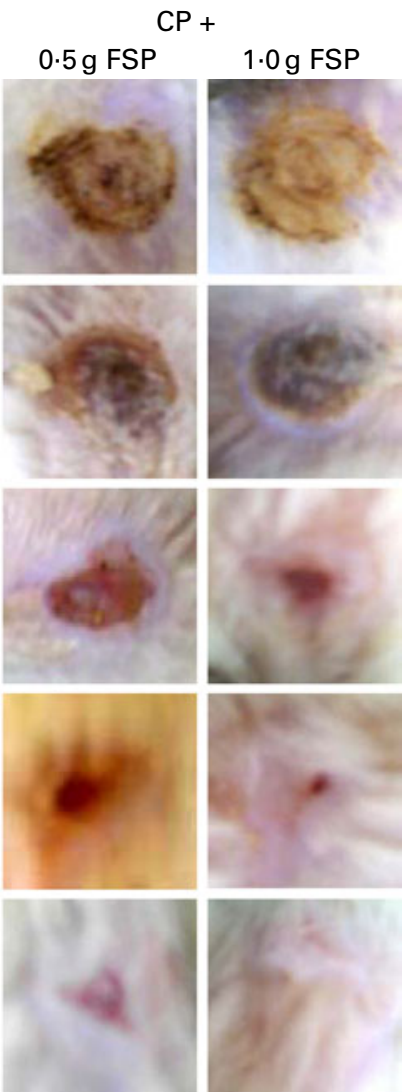

Fig. 4. Modulatory effects of fenugreek seed powder (FSP) on the skin-burning healing process in the healthy and immunosuppressed rat models. Photos were taken at $1,8,18,26$ and $33 \mathrm{~d}$ after burning the skin (a-e, respectively). $\mathrm{CP}$, cyclophosphamide. (A colour version of this figure can be found online at journals.cambridge.org/bjn).

cell volume and blood indices were not significantly changed $(P>0.05)$ in the healthy rats that received either the low or high dose of FSP compared with the healthy control animals (data not shown). The only deleterious effect detected in the present study was the significant increase $(18 \%, P<0.05)$ in the clotting time in rats that received the high dose of FSP only compared with the healthy control animals (data not shown). All of these results indicated that FSP doses used in the present study were considered safe in general.

\section{Discussion}

Rats treated with alloxan (a pancreatic $\beta$-cell cytotoxin) and rats fed a hypercholesterolaemic diet display most signs of the metabolic syndrome including hyperglycaemia and dyslipidaemia, which mimic those developed in human hypoinsulinaemia or type 1 diabetes and insulin resistance or type 2 diabetes, respectively ${ }^{(8)}$. The present study showed that FSP significantly alleviated $(P<0 \cdot 01-0 \cdot 001)$ most signs of the metabolic syndrome including hyperglycaemia, hyperlipidaemia (hypercholesterolaemia and hypertriacylglycerolaemia), elevation in atherogenic indices and impairment of liver functions resulting from experimentally induced type 1 diabetes and obesity in male albino rats by alloxan and a hypercholesterolaemic diet, respectively (Tables 1 and 2). These beneficial effects of FSP were dose dependent in most cases. The hypolipidaemic effect and the marked decrease in atherogenic indices shown in the present study by FSP in rats treated with alloxan or fed a hypercholesterolaemic diet (Table 2) may reduce the incidence of atherosclerosis in diabetic and obese patients.

Fenugreek seeds are used as a condiment, as a supplement to wheat and maize flour for bread making and as a constituent of the daily diet of the general population in many countries ${ }^{(11)}$. They contain 58\% carbohydrates (mainly a water-soluble galactomannan), $23-26 \%$ protein, $6-7 \%$ fat (mainly PUFA), $5-6 \%$ saponins and $2-3 \%$ alkaloids such as trigonelline ${ }^{(14,26)}$. Soluble dietary fibres of fenugreek seeds delay carbohydrate digestion and absorption by slowing gastric emptying, inhibiting the activity of intestinal disaccharidases and brush-border membrane glucose carriers, increasing gastrointestinal motility and enhancing insulin action ${ }^{(13,26,27)}$. In addition, the alcoholic extract of fenugreek seeds was found to suppress glucose absorption and hepatic gluconeogenesis by inhibiting the activity of intestinal brush-border $\alpha$-amylase (that digests starch and dextrin) and glucose-6-phosphatase, respectively, and to increase glucose metabolism by increasing the activity of glucose-6-phosphate dehydrogenase, a hexose monophosphate pathway shunt enzyme ${ }^{(28)}$. 
Moreover, the amino acid, 4-hydroxyisoleucine, of fenugreek seed has insulin secretagogue activity ${ }^{(29)}$. Diosgenin (a major aglycone of saponins) improves glucose metabolism and dyslipidaemia in obesity-related diabetes by inhibiting inflammation in adipose tissues and decreasing the size of adipocytes, which are inversely related to insulin sensitivity, through stimulating adiponectin expression in adipose tissues and promoting adipocyte differentiation, respectively ${ }^{(30)}$. In addition, sapogenins, such as diosgenin, show lipid-lowering activity by increasing biliary cholesterol excretion ${ }^{(31)}$. Fenugreek seeds are also rich in flavonoids $(>100 \mathrm{mg} / \mathrm{g})$, such as quercetin and naringenin ${ }^{(14)}$. Quercetin prolongs insulin signalling by improving tyrosine phosphorylation through the activation and inhibition of protein tyrosine kinases and protein tyrosine phosphatases, respectively ${ }^{(32)}$. Naringenin inhibits 3-hydroxy-3-methylglutaryl-CoA reductase and cholesterol acyltransferase, a rate-limiting enzyme in cholesterol biosynthesis and a key enzyme involved in the esterification and absorption of cholesterol, secretion of hepatic LDL-cholesterol, and cholesterol accumulation in the arterial wall, respectively ${ }^{(14)}$. All of the aforementioned reports may explain the anti-metabolic syndrome activity of FSP shown in the present study.

An interesting finding in the present study was that FSP significantly modulated $(P<0.001)$ both the severe decrease (31\%) and increase (25\%) in body weight induced by alloxan and a hypercholesterolaemic diet, respectively (Table 1), without changing food consumption (data not shown). These results suggest that FSP may have a positive anabolic effect in the type 1 diabetic rats by decreasing the degeneration of the adipocytes and muscle tissues through improving glucose metabolism. Moreover, the significant decrease $(P<0.001)$ in bodyweight gain in addition to the liver-weight:body-weight ratio shown in the obese rats that received the high dose of FSP may be due to a reduction in body and liver fats by improving lipid metabolism, increasing energy expenditure and affecting fat absorption and excretion. Thus, the results of the present study suggest a direct effect of FSP on the improvement of metabolism rather than affecting food intake.

Recently, attention has been focused on the relationship between production of free radicals, especially reactive oxygen species, and the pathogenesis and progression of many diseases including diabetes mellitus. Mechanisms that contribute to the formation of free radicals in diabetes mellitus may include metabolic stress resulting from changes in energy metabolism, inflammatory mediators and impaired antioxidant defence mechanisms ${ }^{(33)}$. There is a strong positive correlation between the production of reactive oxygen species in addition to a decrease in endogenous radical scavengers and hyperglycaemia, dyslipidaemia, membrane lipid peroxidation and cellular injury caused by alloxan and a hypercholesterolaemic $\operatorname{diet}^{(14,34-36)}$. GSH, the first-line defence against lipid peroxidation, is an essential electron donor to glutathione peroxidases in the reduction of hydroperoxides and serves as a nucleophilic co-substrate to glutathione-S-transferases in the detoxification of xenobiotics ${ }^{(37)}$. The reduction of cellular toxicity markers (serum alanine aminotransferase, ASAT and ALP activities) and the elevation in the liver GSH level shown in the type 1 diabetic and obese rats by consuming FSP (Table 1) indicated that the chemical components of FSP prevented hepatocellular damage by stabilising the integrity of the cell membrane, keeping the membrane intact and the enzymes enclosed, through scavenging free radicals. This antioxidant activity of fenugreek seeds may be attributed to the active H-donating ability of the hydroxyl substitutions of their flavonoids ${ }^{(38)}$. The modulatory effect of FSP on the liver GSH level was either partial or complete in the type 1 diabetic or obese rat model, respectively. Moreover, the liver GSH level in the obese rats that received the high dose of FSP exceeded that in the healthy control animals. These results may explain why FSP was more efficient in alleviating the signs of the metabolic syndrome in the obese animals (over 9\%) than in the type 1 diabetic animals.

$\mathrm{CP}$ is a commonly used anti-cancer drug that causes toxicity by its reactive metabolites such as acrolein and phosphoramide mustard, which induce oxygen radical formation and immunosuppression, respectively ${ }^{(39)}$. The present study showed, probably for the first time, that FSP (especially the high dose) completely modulated the immunosuppressive activity of CP including leucopenia (which was mainly due to neutropenia and lymphopenia), the decrease in weights and cellularity of lymphoid organs, the deficiency in humoral and cellular immune responses (as indicated by the decrease in the serum $\gamma$-globulin level and delayed type of hypersensitivity response, respectively), and the delay in the skin-burning healing process (Figs. 1-4). Therefore, FSP may reduce the incidence of infections in cancer patients treated with such chemotherapeutic agents. The significant increase $(P<0.001)$ induced by FSP in bone marrow cellularity in the CP-treated rats (Fig. 2(b)) indicated a stimulatory effect of fenugreek seeds on bone marrow haematopoietic stem cells, which may be, in part, due to the considerable amount of Fe found in fenugreek seeds ${ }^{(11)}$. The increase in the weight of lymphoid organs (thymus and spleen) induced by FSP in the CP-treated animals was concomitant with the increase in their cellularity (Fig. 2). Another study found that $100 \mathrm{mg} / \mathrm{kg}$ body weight of an Indian fenugreek aqueous extract was the optimum dose that induced a stimulatory effect on immune functions in healthy mice, and this immunostimulant activity of fenugreek decreased by increasing the dose ${ }^{(11)}$. In the present study, the immunostimulant activity of Egyptian fenugreek seeds increased by increasing the dose from 0.5 to $1.0 \mathrm{~g} / \mathrm{kg}$ body weight in immunosuppressed rats. The immunostimulant activity of fenugreek seeds may be due to the mitogenic and antioxidant properties of its constituents, which stimulate the 
immunocompetent cells ${ }^{(11,40)}$. Saponins, fibres and flavonoids of fenugreek seeds are the most likely components responsible for their immunostimulant activity ${ }^{(11)}$.

There is an overall decrease in the glutathione content in various tissues as a result of $\mathrm{CP}$ treatment ${ }^{(39)}$. Acrolein induces the GSH depletion by interacting with its amino acid, cysteine ${ }^{(41)}$. Restoration of the GSH level by FSP, as shown in the present study in the type 1 diabetic and obese rats, may play an important role in reversing $\mathrm{CP}$ induced immunosuppression. $\mathrm{CP}$ is known to result in hypercholesterolaemia and hypertriacylglycerolaemia, which are well-known risk factors in $\mathrm{CVD}^{(6)}$. The hypolipidaemic activity of FSP shown in the present study may protect against hyperlipidaemic cardiomyopathy in $\mathrm{CP}$-treated cancer patients. Another interesting finding in the present study was that the modulatory effects of FSP on CP-induced immunosuppression exceeded that on alloxan-induced type 1 diabetes and cholesterol-induced obesity by 24 and $15 \%$, respectively. This result indicated that the immunostimulant activity of fenugreek seeds exceeded their anti-metabolic syndrome activity. The FSP doses used in the present study were considered safe in general, despite the mild increase in the clotting time shown in rats that received the high dose of FSP (data not shown), which may be due to the coumarin derivatives existing in fenugreek seeds ${ }^{(31)}$. In conclusion, fenugreek seeds may be useful not only as a dietary adjunct for the control of the metabolic syndrome in diabetic and obese patients, but also as an immunostimulant in immunocompromised patients such as those under chemotherapeutic interventions.

\section{Acknowledgements}

The present study received no specific grant from any funding agency in the public, commercial or not-for-profit sectors. N. M. E.-B. and G. R. planned the study, designed all the experiments, summarised, discussed and interpreted the results, and drafted the manuscript. H. F. A. E.-K. carried out the experiments and performed the statistical analysis with assistance from G. R. The authors have no potential financial conflict of interest.

\section{References}

1. Lefebvre P (2005) Diabetes yesterday, today and tomorrow. The action of the International Diabetes Federation. Rev Med Liege 60, 273-277.

2. Rossner S (2002) Obesity: the disease of the twenty-first century. Int J Obes Relat Metab Disord 26, Suppl. 4, S2-S4.

3. Knuiman MW, Hung J, Divitini ML, et al. (2009) Utility of the metabolic syndrome and its components in the prediction of incident cardiovascular disease: a prospective cohort study. Eur J Cardiovasc Prev Rehabil 16, 235-241.

4. de Jonge ME, Huitema AD, Rodenhuis S, et al. (2005) Clinical pharmacokinetics of cyclophosphamide. Clin Pharmacokinet 44, 1135-1164.
5. Bin-Hafeez B, Ahmad I, Haque R, et al. (2001) Protective effect of Cassia occidentalis L. on cyclophosphamideinduced suppression of humoral immunity in mice. J Ethnopharmacol 75, 13-18.

6. Mythili Y, Sudharsan PT, Sudhahar V, et al. (2006) Protective effect of DL-alpha-lipoic acid on cyclophosphamide induced hyperlipidemic cardiomyopathy. Eur J Pharmacol 543, 92-96.

7. Ramadan G, El-Beih NM \& Badr GM (2006) Modulatory effects of aged garlic extract in comparison with fresh garlic extract: II - on induced immunosuppression and systemic anaphylaxis in male albino rats. Egypt J Zool 46, 265-283.

8. Ramadan G, El-Beih NM \& Abd El-Ghffar EA (2009) Modulatory effects of black $v$. green tea aqueous extract on hyperglycaemia, hyperlipidaemia and liver dysfunction in diabetic and obese rat models. Br J Nutr 102, 1611-1619.

9. Mowla A, Alauddin M, Rahman MA, et al. (2009) Antihyperglycemic effect of Trigonella foenum-graecum (fenugreek) seed extract in alloxan-induced diabetic rats and its use in diabetes mellitus: a brief qualitative phytochemical and acute toxicity test on the extract. Afr J Tradit Complement Altern Med 6, 255-261.

10. Seo YJ, Gweon OC, Lee YM, et al. (2009) Effect of garlic and aged black garlic on hyperglycemia and dyslipidemia in animal model of type 2 diabetes mellitus. J Food Sci Nutr 14, 1-7.

11. Bin-Hafeez B, Haque R, Parvez S, et al. (2003) Immunomodulatory effects of fenugreek (Trigonella foenum graecum L.) extract in mice. Int Immunopharmacol 3, 257-265.

12. Handa T, Yamaguchi K, Sono Y, et al. (2005) Effects of fenugreek seed extract in obese mice fed a high-fat diet. Biosci Biotechnol Biochem 69, 1186-1188.

13. Hannan JM, Ali L, Rokeya B, et al. (2007) Soluble dietary fibre fraction of Trigonella foenum-graecum (fenugreek) seed improves glucose homeostasis in animal models of type 1 and type 2 diabetes by delaying carbohydrate digestion and absorption, and enhancing insulin action. $\mathrm{Br} J$ Nutr 97, 514-521.

14. Belguith-Hadriche O, Bouaziz M, Jamoussi K, et al. (2010) Lipid-lowering and antioxidant effects of an ethyl acetate extract of fenugreek seeds in high-cholesterol-fed rats. J Agric Food Chem 58, 2116-2122.

15. Ellman GL (1959) Tissue sulfhydryl groups. Arch Biochem Biophys 82, 70-77.

16. Trinder P (1969) Determination of glucose in blood using glucose oxidase with an alternative oxygen acceptor. Ann Clin Biochem 6, 24-27.

17. Knight JA, Anderson S \& Rawle JM (1972) Chemical basis of the sulfo-phospho-vanillin reaction for estimating total serum lipids. Clin Chem 18, 199-202.

18. Allain CC, Poon LS, Chan CS, et al. (1974) Enzymatic determination of total serum cholesterol. Clin Chem 20, 470-475.

19. McGowan MW, Artiss JD, Strandbergh DR, et al. (1983) A peroxidase-coupled method for the colorimetric determination of serum triglycerides. Clin Chem 29, 538-542.

20. Grove TH (1979) Effect of reagent $\mathrm{pH}$ on determination of high-density lipoprotein cholesterol by precipitation with sodium phosphotungstate-magnesium. Clin Chem 25, 560-564.

21. Friedewald WT, Levy RI \& Fredrickson DS (1972) Estimation of the concentration of low-density lipoprotein cholesterol in plasma, without use of the preparative ultracentrifuge. Clin Chem 18, 499-502.

22. Reitman S \& Frankel S (1957) A colorimetric method for the determination of serum glutamic oxalacetic and glutamic pyruvic transaminases. Am J Clin Pathol 28, 56-63. 
23. Wenger C (1984) Alkaline phosphatase. In Clinical Chemistry, pp. 1094-1098 [A Kaplan, editor]. St Louis, MO: Mosby.

24. Harding KG, Morris HL \& Patel GK (2002) Science, medicine and the future: healing chronic wounds. BMJ 324, 160-163.

25. Turner JR \& Thayer JF (editors) (2001) Introduction to Analysis of Variance: Design, Analysis and Interpretation. Thousand Oaks, CA: Sage Publications.

26. Srichamroen A, Thomson AB, Field CJ, et al. (2009) In vitro intestinal glucose uptake is inhibited by galactomannan from Canadian fenugreek seed (Trigonella foenum graecum L.) in genetically lean and obese rats. Nutr Res 29, 49-54

27. Mathern JR, Raatz SK, Thomas W, et al. (2009) Effect of fenugreek fiber on satiety, blood glucose and insulin response and energy intake in obese subjects. Phytother Res 23, 1543-1548.

28. Gad MZ, El-Sawalhi MM, Ismail MF, et al. (2006) Biochemical study of the anti-diabetic action of the Egyptian plants fenugreek and balanites. Mol Cell Biochem 281, 173-183.

29. Sauvaire Y, Petit P, Broca C, et al. (1998) 4-Hydroxyisoleucine: a novel amino acid potentiator of insulin secretion. Diabetes 47, 206-210.

30. Uemura T, Hirai S, Mizoguchi N, et al. (2010) Diosgenin present in fenugreek improves glucose metabolism by promoting adipocyte differentiation and inhibiting inflammation in adipose tissues. Mol Nutr Food Res 54, 1596-1608.

31. Basch E, Ulbricht C, Kuo G, et al. (2003) Therapeutic applications of fenugreek. Altern Med Rev 8, 20-27.
32. Kannappan S \& Anuradha CV (2009) Insulin sensitizing actions of fenugreek seed polyphenols, quercetin \& metformin in a rat model. Indian J Med Res 129, 401-408.

33. Anwar MM \& Meki AR (2003) Oxidative stress in streptozotocin-induced diabetic rats: effects of garlic oil and melatonin. Comp Biochem Physiol A Mol Integr Physiol 135, 539-547.

34. Szkudelski T (2001) The mechanism of alloxan and streptozotocin action in B cells of the rat pancreas. Physiol Res $\mathbf{5 0}$, 537-546.

35. Sabu MC, Smitha K \& Kuttan R (2002) Anti-diabetic activity of green tea polyphenols and their role in reducing oxidative stress in experimental diabetes. J Ethnopharmacol 83, 109-116.

36. Czako L, Szabolcs A, Vajda A, et al. (2007) Hyperlipidemia induced by a cholesterol-rich diet aggravates necrotizing pancreatitis in rats. Eur J Pharmacol 572, 74-81.

37. Barber DA \& Harris SR (1994) Oxygen free radicals and antioxidants: a review. Am Pharm NS34, 26-35.

38. Kaviarasan S, Vijayalakshmi K \& Anuradha CV (2004) Polyphenol-rich extract of fenugreek seeds protect erythrocytes from oxidative damage. Plant Foods Hum Nutr 59, $143-147$.

39. Bhatia K, Kaur M, Atif F, et al. (2006) Aqueous extract of Trigonella foenum-graecum $\mathrm{L}$. ameliorates additive urotoxicity of buthionine sulfoximine and cyclophosphamide in mice. Food Chem Toxicol 44, 1744-1750.

40. Devasagayam TP \& Sainis KB (2002) Immune system and antioxidants, especially those derived from Indian medicinal plants. Indian J Exp Biol 40, 639-655.

41. Kehrer JP \& Biswal SS (2000) The molecular effects of acrolein. Toxicol Sci 57, 6-15. 